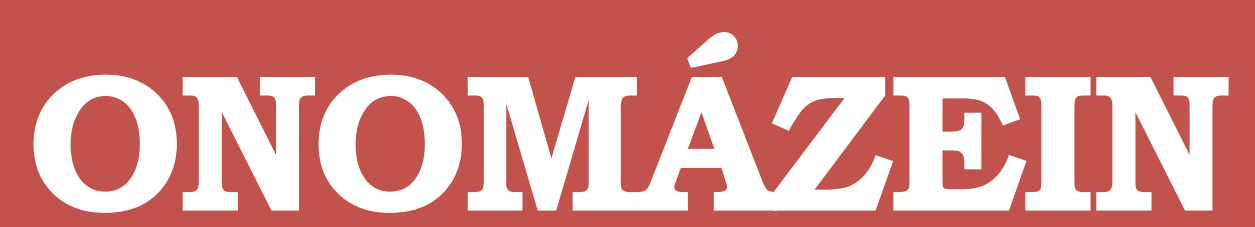

Revista de lingüística, filología y traducción
PONTIFICIA UNIVERSIDAD CATÓLICA DE CHILE FACULTAD DE LETRAS

\title{
Eventos de movimiento en pima bajo
}

Motion events in Pima Bajo

\section{Zarina Estrada Fernández}

Universidad de Sonora

México

\section{Abigail Dariana Anduaga Coello \\ Universidad de Sonora \\ México}

ONOMÁZEIN 52 (junio de 2021): 19-35

DOI: 10.7764/onomazein.52.03

ISSN: $0718-5758$

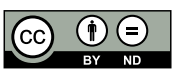

Zarina Estrada Fernández: Departamento de Letras y Lingüística, Universidad de Sonora, México. | E-mail: zarinaef@gmail.com

Abigail Dariana Anduaga Coello: Departamento de Letras y Lingüística, Universidad de Sonora, México.

| E-mail: abigailanduaga@gmail.com 


\section{Resumen}

El presente estudio representa un primer acercamiento a las construcciones que expresan eventos de movimiento en pima bajo, lengua yuto-azteca hablada en el noroeste de México. La investigación explora, desde una perspectiva basada en el uso, las estrategias que tiene esta lengua para la expresión de eventos de movimiento y cómo se codifican. El análisis permite proponer que en esta lengua las nociones de movimiento y trayectoria se encuentran lexicalizadas como Marco-Verbal, pero, además, se consigue constatar eventos con MarcoSatelital, Equipolente y un Sistema Paralelo, lo cual impide que la lengua sea caracterizada tipológicamente como un todo; en su lugar, es conveniente hablar de tipos de construcciones. El estudio alude a lo observado en otras dos lenguas yuto-aztecas: tepehuano del sur y yaqui.

Palabras clave: eventos de movimiento; estudio basado en el uso; lenguas yuto-aztecas; pima bajo; marco verbal.

\section{Abstract}

The present study represents a first approach to the constructions that express motion events in Pima Bajo, a Uto-Aztecan language spoken in northwest Mexico. This contribution explores, from a usage-based perspective, the strategies that this language has for the expression of motion events, and how they are encoded. The analysis allows us to propose that in this language the notions of motion and trajectory appear lexicalized as a Verbal-frame, but constructions also exist which express motion events, with Satellite-frame, Equipollent, and a Parallel system, which prevents the language from being characterized typologically as a whole; instead, it is better to speak of types of constructions. The study makes also reference to what is observed in two other Uto-Aztecan languages: Southern Tepehuan and Yaqui.

Keywords: motion events; usage-based approach; Uto-Aztecan languages; Pima Bajo; VerbFrame. 


\section{Introducción}

Las construcciones con verbos de movimiento han sido foco de atención de múltiples estudios en diferentes lenguas. El objetivo primordial de la mayor parte de estos ha sido verificar las propuestas de Talmy (1985, 2007, principalmente) en torno a la tipología de lenguas de "Marco-Verbal” y "Marco-Satelital”, posteriormente enriquecida por Slobin (2004), al añadir el llamado "Marco-Equipolente".

El presente trabajo desarrolla, por primera ocasión, un análisis de los eventos de movimiento en pima bajo, lengua yuto-azteca de la rama tepimana que se habla en el noroeste de México. El análisis se fundamenta en materiales diversos, principalmente, corpus discursivo (Escalante y Estrada, 1993), cuestionarios centrados en la documentación de eventos de movimiento (cuatro cuestionarios), así como materiales lingüísticos de obras cuyo objetivo es la documentación de diversos tipos de construcciones (Estrada, 1998).

El propósito central es mostrar evidencias de cómo los eventos de movimiento en pima bajo responden a las propuestas de Talmy $(1985,2007)$ y Slobin (2004); específicamente, en cuanto a los patrones de lexicalización que involucran los distintos componentes semánticos que ocurren en la expresión de algún evento de movimiento. Este último, a su vez, puede ser simple, dígase expresado por un solo verbo en una construcción mono-clausal, o complejo, si se codifica por más de un verbo en una construcción multi-verbal. Una segunda aportación es mostrar, desde una perspectiva basada en el uso, la frecuencia de eventos de movimiento que ocurren en el corpus discursivo analizado, ya que estas muestras espontáneas (18 narraciones con 383 instancias de eventos de movimiento) parecen favorecer la preferencia por el Marco-Verbal².

La comunicación de un evento de movimiento se codifica, prototípicamente, por medio de una cláusula simple de un solo argumento, cuyo referente corresponde a la entidad u objeto que se desplaza o cambia de posición según lo indique el significado del verbo de movimiento. De acuerdo con Talmy (1985, 2000, 2007) y Slobin (2004), las construcciones que expresan eventos de movimiento conforman un abanico diverso que difieren entre sí, en relación con la codificación de los elementos que expresan nociones espaciales o locativas, direccionales e incluso de manera. Estos distintos tipos de construcciones son los que dieron lugar a la tipología mencionada en el primer párrafo de esta sección, dígase,

1 Guerrero (2014) incluye dos estudios que tratan de dos lenguas yuto-aztecas: yaqui, desarrollado por la editora de la obra, y o'dam, más conocido como tepehuano del sureste, de García Salido.

2 Este enfoque valora los estudios fundamentados en el análisis de corpus, los cuales son de extrema utilidad sobre todo en lenguas con un número reducido de hablantes (menos de 500) como el pima bajo. 
Marco-Verbal, Marco-Satelital y Marco-Equipolente. Esta tipología enfatiza que las nociones espaciales o direccionales se pueden encontrar: (i) expresadas en el verbo, Marco-Verbal; (ii) a manera de adjuntos o satélites, Marco-Satelital, o bien (iii) codificadas mediante construcciones seriales, Marco-Equipolente. Sin embargo, en la literatura sobre el tema, fueron añadidos (iv) eventos de movimiento que muestran dos posibilidades, combinando dos de los marcos anteriores, lo que se ha denominado Sistema-Paralelo. Inicialmente, esta tipología se aplicó heurísticamente, es decir, para toda la lengua, pero a partir de la exploración de los distintos eventos de movimiento en las diferentes lenguas del mundo, se ha logrado afirmar que, aunque las lenguas tengan una tendencia preferencial hacia uno de estos tipos, la variedad de comportamientos de los distintos verbos valida que algunas de ellas exhiban más de uno de estos marcos.

En este trabajo mostramos, desde una perspectiva basada en el uso, que los eventos de movimiento en pima bajo manifiestan, en general, una fuerte tendencia hacia el Marco-Verbal, es decir, codifican inherentemente la trayectoria, dígase, que esa noción se encuentra lexicalizada en la forma verbal. Sin embargo, el análisis también indica que algunos verbos pueden mostrar, alternativamente, el Marco-Satelital, o bien el Marco-Equipolente, lo que indicaría que este grupo limitado de verbos cuenta con la posibilidad de un Sistema-Paralelo.

Después de esta introducción, la sección 2 presenta varias de las nociones fundamentales para explicar los eventos de movimiento, la sección 3 discute los resultados observados en pima bajo, la sección 4 muestra los resultados encontrados basados en el uso y finalmente la sección 5 presenta las conclusiones.

\section{Marco teórico}

Los estudios sobre los eventos de movimiento proponen que el verbo es el elemento central que expresa la noción de movimiento, dígase el cambio de locación de una entidad u objeto, lo que conlleva, aunque no obligatoriamente, el desplazamiento de ese referente en un determinado lugar o espacio y a lo largo de una trayectoria o dirección. En su propuesta inicial de análisis de los eventos de movimiento, Talmy (1985) propone la representación lingüística de una estructura conceptual (EC) para explicar así los elementos semánticos involucrados en la expresión de este tipo de eventos. Asimismo, aunque inicialmente plantea tres componentes semánticos, posteriormente incluye cuatro o cinco de ellos (Talmy, 1972, 1975, 1985) según se incluya o no la manera del movimiento. Estos componentes son:

(1) a. MOVIMIENTO (MOVE)

b. FIGURA (FIGURE)

C. FONDO (GROUND)

d. TRAYECTORIA (PATH)

e. MANERA (MANNER) 
Los primeros cuatro componentes se ilustran en el ejemplo en (2):

\begin{tabular}{|c|c|c|c|c|}
\hline (2) FIGURA & & MOVIMIENTO & TRAYECTORIA & FONDO \\
\hline The & pencil & rolled & off & the table \\
\hline DET & lápiz & rodar-pAs & fuera & DET mesa ${ }^{3}$ \\
\hline
\end{tabular}

'El lápiz rodó fuera de la mesa.' (Talmy, 1985: 61)

En el ejemplo (2), the pencil 'el lápiz' representa la figura, ya que es el elemento que experimenta el movimiento y es afectado con un cambio de posición. Asimismo, the table 'la mesa' representa el fondo, como el término que alude al espacio a través del cual se desplaza la figura. El verbo rolled 'rodar', a su vez, codifica el movimiento y también la manera del movimiento, debido a que la entidad se mueve rodando sobre sí misma. Por último, off ‘fuera de’ funciona como satélite de trayectoria; lo mismo ocurre si atendemos a lo que el ejemplo expresa en la traducción al español.

La manera, a su vez, puede expresarse en la forma léxica del verbo de movimiento, como en rolled 'rodar', en (2), o bien como elemento independiente, añadiendo así un quinto componente, como se ilustra en (3), donde la manera se expresa mediante el verbo en gerundio, brincando. Además, se observa que ambos, movimiento y trayectoria, se encuentran en el verbo:

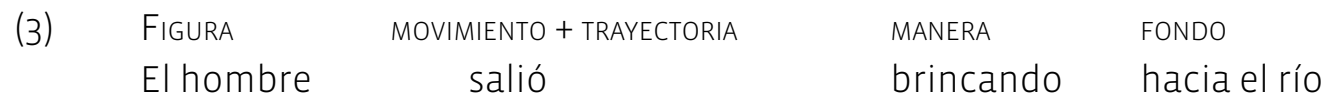

Es precisamente la codificación de la manera como un verbo en gerundio, como en (3), lo que ha hecho que las construcciones con verbos de movimiento entren en la discusión de las Ilamadas construcciones multiverbales. Específicamente Slobin (2004) advierte sobre la diferencia entre lenguas que expresan la manera en el verbo (lenguas de Marco-Verbal) frente a aquellas que la expresan mediante un coverbo o verbo serial (véase también Beavers y otros, 2010: 332), dígase Marco-Equipolente.

De esta manera, los componentes, figura y movimiento, son centrales y obligatorios en toda proposición que exprese un evento de movimiento; el primero se refiere al único argumento de un verbo intransitivo, es decir, el verbo de movimiento, y el segundo al movimiento propiamente dicho, que específicamente denota el contenido semántico del verbo. A su vez, el componente denominado fondo alude al espacio, sitio o lugar sobre el cual se desplaza o

3 Abreviaturas: clas clasificador, conj conjunción, cont continuativo, CUANT Cuantificador, dem demostrativo, DET determinante, DIM diminutivo, DIR direccional, IMPF imperfectivo, INT intensivo, IRR irreal, HAB habitual, Loc locativo, MED media, MD marcador discursivo, NEG negativo, NOM nominativo, NMLz nominalizador, PAS pasado, PART partícula, PFV perfectivo, PL plural, pOS posesivo, POSP posposición, PROB probable, Pros prospectivo, sg singular, sI sujeto idéntico, suJ sujeto, REP reportativo, RDP reduplicación, REM remoto, TEMP temporal, TERM terminativo. 
mueve el referente identificado como figura. El componente fondo se expresa, por lo general, a través de una construcción locativa correspondiente a un argumento oblicuo o adjunto.

Desde esta perspectiva, la interrogante principal en el análisis de los eventos de movimiento alude a las estrategias que las lenguas tienen para la codificación de la trayectoria y la manera. Es justo en la identificación de estas estrategias cuando Talmy (1985) se refiere al fenómeno de la lexicalización. Primero, porque la trayectoria puede estar codificada en el verbo léxico en sí mismo, como en el verbo salir, en (3), o bien ser codificada como un elemento externo al verbo, como en (2). Esta situación, como ya mencionamos, motiva la tipología inicial de Talmy (1985), que propone la existencia de lenguas de Marco-Verbal y Marco-Satelital y que Slobin (2004) enriquece al observar que, de forma similar, (i) la manera puede estar codificada en el verbo como en rolled, donde la manera expresa movimiento sobre sí mismo; (ii) expresada por una forma verbal no finita, como el gerundio brincando, en (3), o bien (iii) ser expresada por medio de otro verbo con el mismo estatus gramatical que el de movimiento, es decir, mediante verbos seriales, lo que denomina Marco-Equipolente, lo que se sintetiza en (4).

(4) Marcos de lexicalización

i. Marco-Verbal. El movimiento y la trayectoria se lexicalizan en la raíz verbal4.

ii. Marco-Satelital. La trayectoria se lexicaliza en un elemento satélite del verbo. Puede ser un clítico, un afijo, una partícula u otros elementos que formen parte de la palabra verbals.

iii. Marco-Equipolente. Los elementos semánticos básicos del evento de movimiento se lexicalizan en dos o más verbos que tienen el mismo estatus gramatical (verbos seriales) $)^{6}$.

Además, en atención al comportamiento diferencial de las lenguas en lo relativo a los marcos de lexicalización, Talmy (2007) propone que se pueden distinguir dos tipos de sistemas de acuerdo con la posibilidad de mostrar más de uno de los marcos descritos en (4). Esto da lugar a lo que se ha llamado Sistema Paralelo y Sistema Dividido. En (5) capturamos esta diferenciación:

(5) Sistemas de lexicalización

i. Sistema Dividido. Cierto tipo de evento muestra un patrón de lexicalización específico de acuerdo con sus características semánticas y en un distinto tipo de evento

4 Algunas lenguas de Marco-Verbal son: francés (Beavers y otros, 2010) y hup (Epps y Neely, 2014), entre otras.

5 Entre las lenguas que se han caracterizado como de Marco-Satelital se encuentran el inglés, alemán y el mandarín (Beavers y otros, 2010), entre otras.

6 Lenguas con Marco-Equipolente: emai (Schaefer, 1986: 181), tailandés (Zlatev y Yangklang, 2004). 
ocurre otro diferente. En este sentido los patrones de lexicalización están en distribución complementaria.

ii. Sistema Paralelo. El mismo evento de movimiento puede expresarse alternativamente por medio de diferentes marcos de lexicalización.

\section{Eventos de movimiento en pima bajo}

El análisis de los eventos de movimiento de pima bajo, que aquí presentamos, consideró, como punto de partida, la organización de ellos de acuerdo con su contenido semántico (Morimoto, 2001: 50): (i) verbos que expresan solamente movimiento, en pima bajo him 'ir' (Move); (ii) eventos de movimiento básico cuyo significado no alude a una trayectoria, por ejemplo, oimar 'andar'; (iii) eventos de movimiento que denotan trayectoria como gii 'caer'; (iv) eventos cuyo significado denota manera del movimiento, como mir 'correr' y da'ia 'volar'; y, finalmente, (v) eventos de movimiento contenido en sí mismo, es decir, sin trayectoria implicada y sin especificar un fondo o locación, como sasa 'sacudir'. Por último, existe la posibilidad de lexicalizar eventos de movimiento como verbos seriales que en conjunto expresan el movimiento y la trayectoria o la manera.

Los resultados de nuestro análisis permiten visualizar al pima bajo como una lengua básicamente de Marco-Verbal. Además, las observaciones fundamentadas en el corpus discursivo —investigación basada en el uso - nos permitieron constatar que todo verbo que mostró un Marco-Satelital o Marco-Equipolente también presentó el de Marco-Verbal, lo cual da lugar a un Sistema Paralelo.

\subsection{Marco-Verbal}

En construcciones de Marco-Verbal existen distintas formas de "fusión" de acuerdo con los tipos de lexicalización. Según lo propuesto por Talmy (2007: 76-88), las distintas fusiones muestran que el verbo puede lexicalizar: (i) movimiento y trayectoria; (ii) movimiento y coevento de manera; (iii) movimiento y figura; y, finalmente, (iv) movimiento y fondo, aunque este último no es tipológicamente común. En pima bajo solamente se atestiguó el primer y segundo tipo, es decir, la fusión en el verbo del movimiento y la trayectoria, y la del movimiento y manera.

En las siguientes secciones, a partir de \$3.1.1 y hasta \$3.1.4, se presentan los tipos de fusiones de Marco-Verbal del pima bajo.

\subsubsection{Eventos de movimiento que no denotan trayectoria}

En (6) se ilustra una expresión donde el verbo oimar 'andar' denota movimiento con desplazamiento, sin codificar la trayectoria ni la manera como se lleva a cabo el movimiento. 


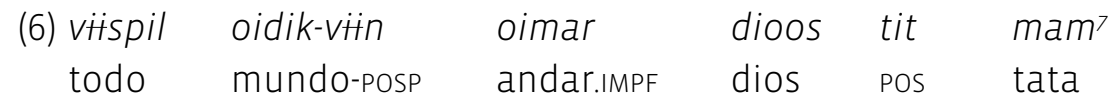

'Así anduvo Nuestro Tata Dios (con su perro) por todo el mundo.'

(Escalante y Estrada, 1993: 188, línea 19)

\subsubsection{Eventos de movimiento que denotan trayectoria}

Estos eventos prototípicamente se lexicalizan con Marco-Verbal, donde la trayectoria y el movimiento se expresan en el verbo. En (7) se expresa un evento de movimiento en el que el verbo głi 'caer' denota trayectoria, de un punto superior a uno inferior, un caso típico de Marco-Verbal. Así, tanto el movimiento como la trayectoria se encuentran lexicalizados en el verbo gii 'caer'. A su vez, la frase posposicional aki-tam 'en el río' codifica el fondo, es decir, un punto fijo en el espacio o meta hasta donde se llevó a cabo el movimiento de la figura, en este caso Huaan.
(7) huaan gij aki-tam
Juan caer.pFv río-Loc
'Juan se cayó en el río.'

(Estrada, 1998: 112, línea 467)

La expresión (8) ratifica el Marco-Verbal, ya que el verbo vaak 'entrar' codifica el movimiento y la trayectoria, aunque, a diferencia de (7), en (8) se añade un co-evento de manera. El co-evento de manera ocurre con el verbo mir 'correr' con un sufijo nominalizador -kam, en forma no finita.

(8) okis mir-kam kosin-tam vaak

mujer correr-nmLz cocina-Loc entrar.pFv

'La mujer entró corriendo a la cocina.'

(Elicitación) ${ }^{8}$

\subsubsection{Eventos de movimiento con manera}

Estos eventos codifican en la forma verbal el movimiento y la manera. En (9) y (10) se ilustran dos expresiones de movimiento que lexicalizan la manera en la forma verbal mir 'correr' y da'ia 'volar', lo que indica un Marco-Verbal.
(9) $a m$
$a=$ tots $-\dot{+r}$
$k=i t$
mir
LOC
MED=RDP aSUStar-TERM
CONJ-SI
correr.pFv
'Allá lo asustaron y entonces corrió.'
(Escalante y Estrada, 1993: 143, línea 15)

7 Los datos de Escalante y Estrada (1993) y Estrada (1998) han sido adaptados en su representación escrita y glosas a la literatura posterior sobre esta lengua, especialmente Estrada Fernández (2014). La traducción corresponde al significado literal.

8 Slobin (1996) señala que en el discurso espontáneo las lenguas de Marco-Verbal predominante tienden a omitir la manera del movimiento, razón por la cual elegimos un ejemplo elicitado. 
(10) ig u’uhug si' mikad da'ia

DEM pájaro INT lejos volar.ProB

'Ese pájaro puede volar mucho.'

(Estrada, 1998: 90, línea 267)

\subsubsection{Eventos de movimiento sin desplazamiento}

Estos eventos contienen un verbo que denota el movimiento, pero no un cambio de posición de la figura, ni especifican fondo o locación. En el corpus analizado se encontraron dos verbos con estas características semánticas: sasa ‘sacudir' y ho'ini 'balancearse'. Véase en (11) que el verbo, sasa 'sacudir', muestra el Marco-Verbal; además, se expresa varias veces, como resultado de la oralidad y contexto pragmático que enfatiza la iteratividad del evento de movimiento.

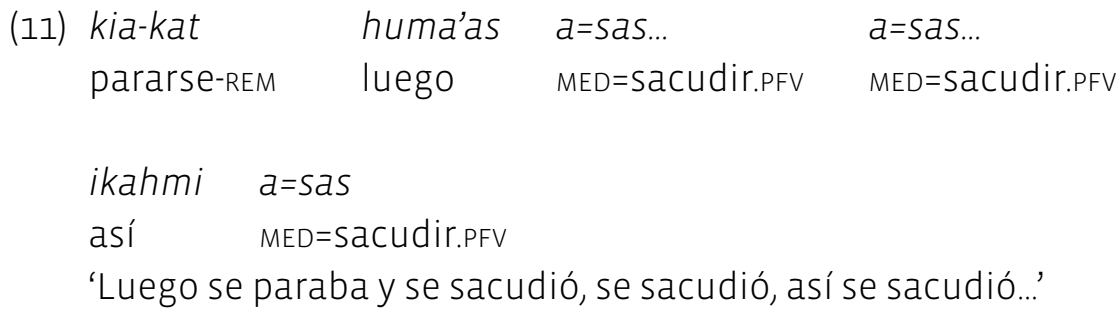

(Escalante y Estrada, 1993: 211, línea 5)

En (12), el verbo hoi'ni 'balancearse' denota un evento de movimiento sin desplazamiento, ya que la figura no muestra un cambio de posición, y solamente se mueve de cierta manera, sin expresión de la trayectoria, lo que indica un Marco-Verbal.

$\begin{array}{lllll}\text { (12) si } & \text { mu'i obig, mu'i as } & a=h o i ' n i \\ \text { INT } & \text { CUANT gente INT } & \text { REP } & \text { MED=mover.IMPF }\end{array}$

'Mucha gente se movía.'

(Escalante y Estrada, 1993: 174, línea 38)

\subsection{Marco-Satelital}

Los eventos de movimiento de Marco-Satelital en pima bajo se caracterizan en que la trayectoria está codificada mediante elementos adverbiales externos al verbo; tal es el caso de los deícticos am 'distal' (desde el ego) y ab 'proximal' (hacia el ego). En (13) se presenta un caso del verbo him, el cual, al no ocurrir con un fondo determinado, tiene una interpretación de 'ir' (move), es decir, un movimiento desde el centro deíctico ego hacia otro lugar, aunque no se especifica cuál. En (13) la figura es omitida porque fue previamente mencionada en el discurso.

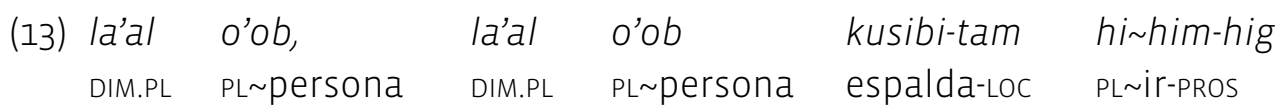

'Iban con niños en la espalda.'

(Escalante y Estrada, 1993: 162, línea 34) 
De acuerdo con Stolz y otros (2014: 56), los principios de predictibilidad y economía permiten que el verbo move tenga una interpretación alativa de movimiento, como el caso de him en pima bajo. De esta manera, si una relación espacial entre una figura y un movimiento es lo suficientemente clara a partir del contexto o el conocimiento del mundo, la expresión de la trayectoria se puede omitir. Lo anterior ha sido constatado por Nakazawa (2006: 288), quien sostiene que la trayectoria de 'venir' está ligada a un fondo determinado, mientras que los verbos de ir indican movimiento o desplazamiento, sin una meta puntual obligatoria.

En (14a) him, acompañado del direccional deíctico am, tiene la interpretación de ir, es decir, movimiento desde el centro deíctico hacia otro lugar lejos del ego. En cambio, him acompañado del deíctico ab, como en en (14b), cambia la orientación de la trayectoria y expresa 'movimiento hacia el ego'.

$\begin{array}{rllll}\text { (14) a. higi } & \text { dioos } & \text { tit-mam } & \text { am } & \text { a-hi-him-va } \\ \text { 3SG.SuJ } & \text { dios } & \text { IPL.NSUJ-padre } & \text { DIR } & \text { MED-PL ir-COMPL }\end{array}$

'Dios Nuestro Padre se fue'

(Escalante y Estrada, 1993: 162, línea 37)

\begin{tabular}{|c|c|c|c|c|c|}
\hline$p s$ & $a b$ & hi-him & higi & $o o b$ & maayk-tam \\
\hline ches & DIR & PL ir.CONT & 3PL & gente & Maycoba-ıoc \\
\hline
\end{tabular}

(Escalante y Estrada, 1993: 80, línea 5)

Otro caso de Marco-Satelital se presenta en (15). Al combinarse la raíz buah 'cargar' con el deíctico distal am se expresa 'movimiento de cargar algo desde el ego hacia otro lugar', es decir, 'Ilevar', como se aprecia en (15a). En cambio, en (15b), la raíz buah 'cargar', en conjunto con el deíctico proximal ab, codifica la trayectoria contraria, dando como resultado 'traer', es decir, movimiento desde un lugar lejano hacia el ego.

(15) a. aan noar himak kareet koa'ag am buah-ka=ti
IsG.Suj comprar.PFv uno carreta leña DIR cargar-EST=SI
'Compré la carreta para llevar la leña.'

(Estrada, 1998: 123 línea 555)

\begin{tabular}{|c|c|c|c|c|c|}
\hline b. $a b$ & $a-b u a ' a$ & $a b$ & yitps & $a b$ & $\dot{t p}$ \\
\hline DIR & MED-cargar.PFV & DIR & Yepachi & DIR & también \\
\hline
\end{tabular}

(Escalante y Estrada, 1993: 72, línea 11)

Es importante señalar que, en el corpus analizado, ningún evento ocurrió exclusivamente con Marco-Satelital, sino que también se codificaron con Marco-Verbal, por lo que deben ser considerados de Sistema-Paralelo. Los ejemplos en (16) ilustran esta posibilidad, donde el evento de movimiento de acercarse al ego, dígase ‘venir', ocurre codificado a través de dos marcos: en (16a), de 
Marco-Verbal con el verbo duv 'venir', que denota el movimiento y la trayectoria, y en (16b), como Marco-Satelital con el verbo him, que expresa solo el movimiento, y la trayectoria está codificada por medio del satélite ab, dando como resultado el evento 'ir hacia el ego', es decir, 'venir'.

(16) Marco-Verbal

$\begin{array}{lll}\text { a. higam } & \text { ki-tam } & \text { duv } \\ \text { 3Pl.suj } & \text { casa-Loc } & \text { venir.PFV } \\ \text { 'Ellos vinieron a la casa.' } & \end{array}$

(Estrada Fernández, 2014: 190)

Marco-Satelital

b. aan im maat-kat ko aap ab him

ISG.SUJ NEG Saber-REM CON 2SG.SUJ DIR ir.CONT

'Yo no sabía que venías.'

(Estrada Fernández, 1998: 89, línea 260)

\subsection{Marco-Equipolente}

Como señalamos anteriormente, Slobin (2004) propuso el Marco-Equipolente para incluir lenguas que expresan el movimiento y la manera a través de verbos seriales, donde los eventos de movimiento que se lexicalizan en dos o más verbos tienen el mismo estatus gramatical. En estos casos no puede atribuirse un Marco-Verbal debido a que la manera no está codificada por medio de un elemento satelital, dígase un verbo no finito, como en el caso del gerundio del español en 'se fue corriendo'. Asimismo, estas construcciones tampoco pueden considerarse de Marco-Satelital, ya que prototípicamente el co-evento de manera se lexicaliza en el verbo, y la trayectoria a manera de satélite.

En (17) se ilustra un evento de movimiento con un co-evento de manera codificado como Marco-Equipolente. En el ejemplo, ocurren los verbos him 'ir' y nor 'caminar', ambos con el sufijo estativo -ak/-ka, aunado a esta propiedad; Ios verbos comparten un mismo sujeto, y el adverbio temporal humas 'luego' tiene alcance amplio sobre ellos; estos rasgos permiten caracterizarlos como verbos seriales.

(17) humas hi-him-ak ${ }^{9}$ nor-ak

luego PL ir-EST caminar-EST

'Luego los dos fueron caminando.'

(Escalante y Estrada, 1993: 176, línea 50)

9 El sufijo -ak corresponde a una variante del estativo - $k a$, que nominaliza verbos cuando estos expresan actividades simultáneas o consecutivas (equivalente a un gerundio según la terminología de lenguas indoeuropeas). 
El ejemplo en (17) difiere del ilustrado anteriormente en (8) - repetido enseguida por conveniencia-, ya que en este último la manera se encuentra codificada con un verbo nominalizado, mir-kam 'correr-nmLz', lo que impide que pueda atribuirse el mismo estatus gramatical a los verbos.

(8) okis mir-kam kosin-tam vaak mujer correr-nmLz cocina-Loc entrar.pFv

'La mujer entró corriendo a la cocina.'

En resumen, aunque los eventos de movimiento del pima bajo tienden a lexicalizar en el verbo el movimiento y la trayectoria, dígase Marco-Verbal, también existe la opción de especificar manera a través de un Marco-Equipolente y trayectoria mediante un Marco-Satelital, en el cual el verbo lexicaliza el evento movimiento y la trayectoria se codifica por medio de deícticos direccionales. Debido a que el mismo evento puede expresarse por medio de distintas estrategias, debe sostenerse que la lengua muestra un Sistema Paralelo.

\section{Perspectiva basada en el uso y prominencia del Marco-Verbal}

En esta sección se discuten los resultados del análisis de la frecuencia de ocurrencia del Marco-Verbal en una serie de muestras discursivas espontáneas. Se han considerado 18 narraciones anecdóticas, históricas y mitológicas que en su total contienen 383 construcciones con verbos de movimiento. En ellas se observaron 22 verbos distintos que expresan eventos de movimiento pertenecientes a cinco clases semánticas basadas en la clasificación de Morimoto (2001: 50): i. movimiento, como him; ii. movimiento sin trayectoria, como los verbos oimar 'andar', pahaloog 'pasear'; iii. movimiento con trayectoria, como tivnia 'bajar', tiisad 'subir'; iv. movimiento con manera, como milia 'correr.sG', voop 'correr.PL', norgia 'caminar', y v. movimiento contenido en sí mismo, como sas 'sacudirse' y ho'ini ‘menearse’, 'balancearse’.

El resultado del análisis de la frecuencia de ocurrencia de los diferentes verbos en el corpus discursivo se organiza en el cuadro 1 de acuerdo con los distintos marcos de lexicalización. En la columna i se enuncian los elementos de los eventos de movimiento que se codifican en el verbo; en la columna 2 se proporcionan los verbos que ocurrieron en el corpus analizado; en la columna 3 se da el número de veces en que el evento de movimiento es el básico, Move; en las columnas 4, 5 y 6 se presenta el número de ocurrencias de cada uno de los verbos del corpus según se lexicalizaron con Marco-Verbal, Marco-Satelital o bien Marco-Equipolente. Finalmente, en la última columna se da el número de ocasiones que apareció cada verbo.

Los resultados del análisis reflejan que him es el prototípico verbo move, es decir, solo codifica el movimiento; asimismo, se observa que el pima bajo privilegia el Marco-Verbal para codificar los elementos semánticos de los eventos de movimiento. Los únicos verbos que mostraron un Marco-Satelital, es decir, aquellos que se allegaron un marcador adverbial, 


\section{CUADRO 1}

Frecuencia de ocurrencia de marcos de lexicalización

\begin{tabular}{|c|c|c|c|c|c|c|}
\hline $\begin{array}{l}\text { LEXICALIZACIÓN } \\
\text { VERBAL }\end{array}$ & VERBO & MOVE & $\begin{array}{c}\text { MARCO } \\
\text { V }\end{array}$ & $\begin{array}{l}\text { MARCO } \\
\mathrm{S}\end{array}$ & $\begin{array}{c}\text { MARCO } \\
\text { E }\end{array}$ & SUBTOTAL \\
\hline movimiento & 1. him 'mover, ir' & 99 & - & 42 & 7 & 148 \\
\hline \multirow{2}{*}{$\begin{array}{l}\text { movimiento sin } \\
\text { trayectoria }\end{array}$} & 2. oimar 'andar' & - & 8 & - & - & 8 \\
\hline & 3. pahalog 'pasear' & - & 3 & - & - & 3 \\
\hline \multirow{11}{*}{$\begin{array}{l}\text { movimiento con } \\
\text { trayectoria }\end{array}$} & 4. tivnia 'bajar' & - & 3 & - & - & 3 \\
\hline & 5. gtia 'caer' & - & 14 & - & - & 14 \\
\hline & 6. vaak'entrar' & - & 5 & - & - & 5 \\
\hline & 7. u’uk'llevar' & - & 16 & - & - & 16 \\
\hline & 8. vaps 'meter' & - & 6 & - & - & 6 \\
\hline & 9. bib 'regresar' & - & 11 & - & - & 11 \\
\hline & 10. vahan 'sacar' & - & 2 & - & - & 2 \\
\hline & 11. vusan 'salir' & - & 37 & - & - & 37 \\
\hline & 12. tisad 'subir' & - & 8 & - & - & 8 \\
\hline & 13. duvia 'venir' & - & 63 & - & - & 63 \\
\hline & 14. oidi 'seguir' & - & 4 & 7 & - & 11 \\
\hline \multirow{6}{*}{$\begin{array}{l}\text { movimiento } \\
\text { con manera }\end{array}$} & 15. norgia 'caminar' & - & 6 & 3 & - & 9 \\
\hline & 16. milia 'correr.sG' & - & 8 & - & - & 8 \\
\hline & 17. komlihia 'nadar' & - & 2 & - & - & 2 \\
\hline & 18. da'ia 'volar' & - & 3 & - & - & 3 \\
\hline & 19. voop 'correr.PL', & - & 5 & - & - & 5 \\
\hline & 20. buah 'cargar'10 & - & 9 & 8 & - & 17 \\
\hline \multirow{4}{*}{$\begin{array}{l}\text { movimiento } \\
\text { contenido en } \\
\text { sí mismo }\end{array}$} & 21. sasa 'sacudir' & - & 1 & - & - & 1 \\
\hline & 22. ho'ini 'moverse' & - & 3 & - & - & 3 \\
\hline & Total & 99 & 217 & 60 & 7 & 383 \\
\hline & Total \% & $26 \%$ & $57 \%$ & $15 \%$ & $2 \%$ & $100 \%$ \\
\hline
\end{tabular}

son him 'ir', buah 'traer', oidi 'seguir' y norgia 'caminar'. Por último, solamente him 'ir' presentó también Marco-Equipolente en algunas construcciones donde el movimiento y la

10 La semántica de este verbo implica inicialmente un evento de cargar un objeto y, luego, el de trasladarlo a algún lugar o fondo a través de una trayectoria. 
manera se expresan mediante verbos seriales. Debido a que todas las construcciones que presentaron un Marco-Satelital cuentan con una estrategia alternativa, se propone entonces un Sistema Paralelo en esta lengua.

En el cuadro 1 se observa que un $26 \%$ de ocurrencias verbales presentaron la raíz him 'mover, ir' como movimiento con desplazamiento (move), cuya interpretación se basa en el hecho de que el movimiento parte del punto deíctico del ego hacia otro punto en el espacio, es decir, 'ir'. Por otra parte, 57\% de las construcciones presentaron un Marco-Verbal, mientras que otras 60, es decir, el 15\%, mostraron un Marco-Satelital, lo que muestra que los verbos identificados con número 1, 14, 15 y 20, en el cuadro 1, exhibieron un Sistema Paralelo. Además, un 2\% del corpus, constituido por solamente construcciones con el verbo him 'mover, ir', mostró un Marco-Equipolente. Desde la perspectiva basada en el uso, estos resultados demuestran que la lengua otorga prominencia de ocurrencia a las construcciones de Marco-Verbal, lo cual seguramente tiene su razón de ser en la temática de los cuentos, ya que en su mayor parte aluden a temas relativos a confrontaciones entre pueblos, viajes y traslados, y en ese proceso narrativo se acude a verbos de movimiento semánticamente más especializados.

\section{Conclusiones}

Este trabajo significó un primer acercamiento a las construcciones que expresan eventos de movimiento en pima bajo. El análisis permitió concluir que en esta lengua las nociones de movimiento y trayectoria se encuentran lexicalizadas en el verbo, es decir, como MarcoVerbal. La discusión de los datos se fundamentó en una perspectiva basada en el uso; así, 11 de los 22 verbos analizados lexicalizaron movimiento y trayectoria en el verbo, y 217 de las 383 construcciones con verbos de movimiento presentaron Marco-Verbal, es decir $57 \%$ de los datos analizados. El trabajo también pudo mostrar que en la lengua se encuentran cuatro verbos que de manera alternativa presentan Marco-Satelital: him 'ir', buah 'traer', oidi 'seguir' y norgia 'caminar', lo cual abre la posibilidad de sostener un Sistema Paralelo.

Los resultados obtenidos contrastan con lo observado en otras lenguas yuto-aztecas del noroeste de México, específicamente el yaqui, y el tepehuano del sureste u o’odam, que, respectivamente, Guerrero (2014) y García (2014), sitúan como de Marco-Satelital.

En (18) se ilustra un ejemplo de yaqui, en el cual el verbo siika 'ir' codifica movimiento y la trayectoria está lexicalizada en el clítico yeu= 'fuera', para indicar en conjunto 'salir'.

(18) Yaqui (Guerrero, 2014: 224)

$\begin{array}{lllll}\text { ba’a-po } & \text { ili } & \text { chu’u-ø } & \text { ket=into } & \text { ama yeu=siika } \\ \text { agua-toc } & \text { pequeño } & \text { perro-nom } & \text { también=mD ahí fuera=ir.sG.PFv }\end{array}$

'Y también el perrito salió del agua.' 
Por su parte, García (2014: 186) coincide en que el tepehuano del sureste también utiliza Marco-Satelital al expresar eventos de movimiento. En (19), el verbo jim 'mover' denota movimiento, mientras que bha- expresa trayectoria 'hacia acá', para indicar 'venir'.

(19) Tepehuano del sureste o’odam (García, 2014: 186)

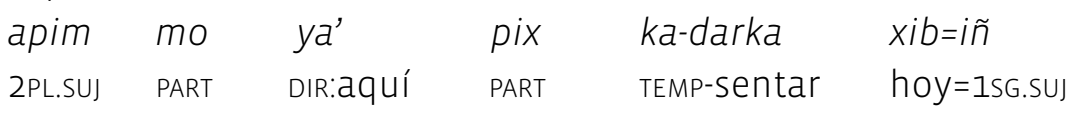

bha-jim

DIR:hacia.acá-mover

'En cuanto a ustedes, aquí se quedan, ahorita vengo.'

Como ya hemos mencionado, las tres lenguas, el yaqui, el tepehuano del sureste y el pima bajo, pertenecen a la familia yuto-azteca, específicamente a la rama taracahita la primera y a la rama tepimana las otras dos. El parentesco genético se observa en que las tres lenguas utilizan el verbo 'ir' como base de construcciones de Marco-Satelital. A su vez, la cercanía genética entre pima bajo y tepehuano del sureste se corrobora porque ambas lenguas - ejemplos (14b-15b) de pima bajo y (19) de tepehuano del sureste- exhiben, respectivamente, una construcción de Marco-Satelital conformada por el deíctico direccional ab + him 'venir' y bha + jim 'venir'. Sin embargo, al lado de esta similitud, también hay comportamientos diferentes entre las tres lenguas, lo cual impide que sean caracterizadas de manera homogénea. En cuanto al yaqui, Guerrero (2014: 244) advierte sobre la gran productividad de la base verbal siime 'ir' combinada con otros elementos como en kom=siime 'ir abajo / bajar', jika=siime 'ir arriba / subir', etc., lo cual, entre otros aspectos, permite considerarla como lengua de MarcoSatelital. Por otra parte, García (2014: 187) señala que en tepehuano del sureste las partículas satelitales de trayectoria que ocurren con el verbo jim 'mover' son obligatorias en todo evento de movimiento de la lengua, por lo que sostiene un Marco-Satelital. Esto contrasta con pima bajo porque en esta última lengua un evento de movimiento con him 'ir' no requiere obligatoriamente de estas partículas, (9).

Por último, recordemos que también fue posible constatar casos de Marco-Equipolente, es decir, construcciones en las cuales los componentes del evento de movimiento están lexicalizados en verbos seriales. En cuanto a este tema, sería necesaria mayor investigación para profundizar en la expresión de eventos de movimiento y manera asociada al mismo sobre todo porque el comportamiento de estos verbos abre la discusión al tema de la compactación o unión de cláusulas. Véase que en ejemplos como el de (20) la construcción es claramente biclausal, lo que se demuestra por la ocurrencia de un marcador de mismo sujeto -ti/-it que apuntala la referencia a dos sujetos idénticos.

(20) kilia duv paan ko'i-m=it
hombre venir-pFv pan comer-conT=SI
'Él vino comiendo pan.'




\section{Bibliografía citada}

Beavers, John, Beth Levin y Shiao Wei Tham, 2010. "The Typology of motion expressions revisited", Journal of Linguistics 46, 331-377.

Epps, Patience, y Kelsey Neely, 2014: "Movimiento y orientación en las construcciones verbales: Una perspectiva amazónica" en Lilián Guerrero (ed.): Movimiento y espacio en lenguas de América, Ciudad de México: Universidad Nacional Autónoma de México, 25-52.

Escalante Hernández, Roberto, y Zarina Estrada Fernández, 1993: Textos y gramática del pima bajo, Hermosillo: Cuadernos de Humanidades. Universidad de Sonora.

Estrada Fernández, Zarina, 1998: Pima bajo de Yepachi, Chihuahua, Archivo de Lenguas Indígenas de México, México: El Colegio de México.

Estrada Fernandez, Zarina, 2014: Gramática de referencia del pima bajo. Vol. I, Hermosillo: Universidad de Sonora.

Garcia, Gabriela, 2014: “La codificación de los verbos de movimiento en o’dam” en Lilián GueRRERo (ed.): Movimiento y espacio en lenguas de América, México: Universidad Nacional Autónoma de México, 179-205.

GuerRero, Lilián, 2014: "Verbos de movimiento y predicados complejos en yaqui" en Lilián GueRRERo (ed.): Movimiento y espacio en lenguas de América, México: Universidad Nacional Autónoma de México, 236-274.

Morimoto, Yuko, 2001: Los verbos de movimiento, Madrid: Visor.

Nakazawa, Tsuneko, 2006: "Motion Event and Deictic Motion Verbs as Path-Conflating Verbs" en Stefan Müller (ed.): Proceedings of the $13^{\text {th }}$ International Conference on Head-Driven Phrase Structure Grammar, Varna, Stanford: CSLI Publications.

SChaefer, Ronald P., 1986: "Lexicalizing directional and nondirectional motion in Emai" en Studies in African Linguistics 16, 57-87.

SLoBiN, Dan I., 1996: "Two ways to travel: Verbs of motion in English and Spanish" en Masayoshi Shibatani y Sandra A. Thompson (eds.): Grammatical constructions: Their form and meaning, Oxford: Oxford University Press, 195-219.

SLoBIN, Dan I., 2004: "The many ways to search for a frog: Linguistic typology and the expression of motion events" en Sven StrömQvist y Ludo Verhoeven (eds.): Relating events in narrative, vol. 2: Typological and contextual perspectives, Mahwah: Lawrence Erlbaum Associates, 219-257. 
Stolz, Thomas, Sander LeStRade y Christel Stolz, 2014: The Crosslinguistics of Zero-Marking of Spatial Relations, Berlín: De Gruyter Mouton.

TALmy, Leonard, 1972: Semantic Structures in English and Atsugewi, Berkeley: University of California. Tesis doctoral.

TALmy, Leonard, 1975: "Semantics and syntax of motion" en John Kimball (ed.): Syntax and Semantics, vol. IV, New York: Academic Press, 181-238.

TALMY, Leonard, 1985: "Lexicalization patterns: Semantic structure in lexical forms" en Timothy SHopen (ed.): Language Typology and Syntactic Description, Vol. 3: Grammatical Categories and the Lexicon, Cambridge: Cambridge University Press, 57-149.

TALmy, Leonard, 2000: Toward a cognitive semantics: Vol. I: Concept Structuring Systems, Cambridge: MIT Press.

TALmy, Leonard, 2007: “Lexical typologies” en Timothy Shopen (ed.): Language Typology and Syntactic Description. Vol. III: Grammatical Categories and the Lexicon, Cambridge: Cambridge University Press, 66-168.

Zlatev, Jordan, y Peerapat YangkLANG, 2004: "A third way to travel: The place of Thai in motionevent typology" en Sven StrömQvist y Ludo Verhoeven (eds.): Relating events in narrative, vol. 2: Typological and contextual perspectives, Mahwah, NJ: Lawrence Erlbaum, 159-190. 